\title{
Investigation of Swelling/Sorption Characteristics of Highly Swollen AAm/AMPS Hydrogels and Semi IPNs with PEG as Biopotential Sorbent
}

\author{
Semiha Kundakci, Erdener Karadağ ${ }^{*}$, Ömer Barış Üzüm \\ Adnan Menderes University, Fen-Edebiyat Faculty, Department of Chemistry, TR-09010, Aydın, Turkey \\ E-mail: ekaradag@adu.edu.tr \\ Received February 20, 2011; revised March 23, 2011; accepted March 30, 2011
}

\begin{abstract}
The aim of this study was to investigate the equilibrium swelling and sorption properties of chemically crosslinked copolymeric hydrogels as biopotential sorbent consisting of acrylamide (AAm) and 2-acrylamido-2methyl-1-propanesulfonic acid (AMPS). Semi-interpenetrating polymer network (semi IPNs) hydrogel, composed of AAm with AMPS as co-monomer, with poly (ethylene glycol) (PEG) and a multifunctional crosslinker such as trimethylolpropane triacrylate (TMPTA) was prepared. AAm/AMPS hydrogels and AAm/AMPS/PEG semi IPNs were synthesized by free radical solution polymerization by using ammonium persulphate (APS)/N,N,N',N'-tetramethylethylenediamine (TEMED) as redox initiating pair. Swelling experiments were performed in water, $0.01 \mathrm{M}$ and $0.03 \mathrm{M}$ aqueous urea solutions at $25^{\circ} \mathrm{C}$, gravimetrically. The hydrogels showed enormous swelling in aqueous urea/water medium and displayed swelling characteristics that were highly depended on the chemical composition of the hydrogels. FTIR spectroscopy was used to identify the presence of different repeating units in the semi IPNs. Some swelling and diffusion characteristics were calculated for different semi IPNs and hydrogels prepared under various formulations. For sorption of cationic dye, Lauths violet into the hydrogels was studied by batch sorption technique at $25^{\circ} \mathrm{C}$. Dye removal capacity, adsorption percentage and partition coefficient of the hydrogels was investigated. Swelling and dye sorption properties of AAm/AMPS hydrogels and AAm/AMPS/PEG semi IPNs were investigated as a function of chemical composition of the hydrogels.
\end{abstract}

Keywords: Swelling, Hydrogel, Interpenetrating Polymer Networks, Acrylamide, 2-acrylamido-2-methyl-1propanesulfonic Acid, Urea, Lauths Violet Sorption

\section{Introduction}

In recent years, polymeric gels are the objects of intensive studies. Highly swollen polymers or copolymers are highly hydrophilic, three-dimensional crosslinked polymeric structures that are able to swell in the aqueous environment. Hydrogels have found numerous uses ranging from daily life applications, mainly due to their high water absorption capacity to the development of new materials for many different purposed applications. Hydrogels are inherently soft, hydrophilic, porous, and elastic polymeric systems. The use of polymer hydrogels as biopotential sorbent or carriers for the removal of the model molecules from aqueous solutions or controlled release studies of them has been continued to attract con- siderable attention in recent years. Hydrogels are polymers in three-dimensional network arrangement, which are able to retain large amount of water. In order to keep the spatial structure, the polymer chains are usually physically or chemically crosslinked. Due to their swelling capacity, hydrogels can be easily rinsed to remove reagents residues. On the other hand, the big water content that makes hydrogels such a special class of materials. The importance of hydrogels in the biomaterial field is justified by some unique characteristics: the elastomeric and soft nature of the hydrogels [1-7].

Crosslinked polymers capable of imbibing large volumes of water have found widespread applications in bioengineering, biomedicine, and food industry and water purification and separation process. Due to its swell- 
ing ability in water, hydrophilicity, biocompatibility, and no toxicity, hydrogels have been utilized in a wide range of biological, medical, pharmaceutical, environmental applications [8-18].

The major environmental problem with colorants is the removal of dyes from effluent waste materials. Untreated effluents from dyestuff production and dyeing mills may be highly colored and thus particularly objectionable if discharged to open waters. Dyeing wastes in some industrials have high color and organic content. Colored waters are also objectionable on aesthetic grounds for drinking and other municipal and agricultural purposes. Effective removal of dyes, in connection with waste water treatment strategy, still remains a major topic of present research. Many methods have been proposed for the removal of dyes, heavy metals and other hazardous materials. Chemical precipitation, membrane extraction, coagulation, complexing, solvent extraction, ion change, and adsorption are some of the commonly used process, but each has its own merits and demerits in its applications. Adsorption procedures are a way of the most widely used for pollutants such as dyes and organic compounds from industrial effluents. Adsorption is a well-known equilibrium separation process. Recently, new effective, efficient and economic methods for water decontamination applications and for separation analytical purposes have been investigated [2-10].

When hydrogels come in contact with aqueous solutions, they adsorb and retain the dissolved substances. For this reason, they have been in several studies such as "for water purification [8], for removal of some dyes from aqueous solutions [9,11,15-18], for removal of toxic metal ions with magnetic hydrogels [10], for uptake of metal ions from aqueous solutions $[10,11,13,18]$, for uptake of uranyl ions from aqueous solutions $[12,18]$ "as water purification agents.

Poly (ethylene glycol), (PEG) is of great interest in numerous biomedical applications for several purposes. PEG is water-soluble and is non-toxic for body immune system. PEG based hydrogels have good biocompatibility. PEG based hydrogel systems have been used at many biotechnological applications $[6,19,20]$.

Some physical properties of hydrogels may be improved by preparing semi-interpenetrating polymer networks (semi IPNs), when the hydrogel network is prepared in the presence of a previously made polymer such as poly (ethylene glycol), polyacrylamide, poly (Nisopropyl acrylamide), poly (vinyl pyrrolidione), poly (vinyl alcohol), or poly (acrylic acid), etc. Water sorption property of hydrogels or semi-interpenetrating polymer networks (semi IPNs) accounts for a great number of biomedical and technological applications such as drug delivery systems, artificial implants, contact lens, en- zyme immobilization, catheters, wound dressings, biosensors, superabsorbents, and etc, [3,6,21,22].

Hydrogels can be used as a composite membrane for various enzymes. For example, for immobilization of Urease enzyme, various composite hydrogel membranes can be used. Urease is a highly specific enzyme. It catalyzes the hydrolysis of urea to ammonium and carbon dioxide. It has been immobilized for analytical and biomedical purposes. One of the major applications of immobilized Urease is the direct removal from blood for detoxification, or in the dialysis regeneration systems of artificial kidney machines. Other applications of immobilized Urease will be in a bioreactor for the conversation of urea present in fertilizer wastewater effluents to ammonia and carbon dioxide or in the food industry for the removal of urea from beverages and foods [4,23-26]. Urea is one of the main toxic wastes in the dialysate solution from hemodialysis. The most effective way of removing urea from aqueous solutions is the utilization of immobilized Urease as no efficient adsorbent is available for urea. On the other hand, urea has a great importance in biological systems. In our previously studies, some papers was reported about swelling characterization of $\gamma$-radiation induced crosslinked acrylamide/crotonic acid and acrylamide/maleic acid hydrogels in urea solutions $[25,26]$.

Polyacrylamide based hydrogels have received considerable attention because of their use in many applications. In our and others previous studies, copolymeric hydrogels of acrylamide with some acidic monomers were prepared by free radical solution polymerization and used in separation and adsorption of some dye molecules [4,6,17,18,27-29].

AMPS received attention in recent years due to its strongly ionizable sulfonate group. Increasing number of ionic groups in the hydrogels is known to increase their swelling capacity. AMPS dissociate completely in the overall $\mathrm{pH}$ range, and therefore, the hydrogels derived from AMPS exhibit $\mathrm{pH}$ independent swelling behavior $[6,9,13,30,31]$.

The present paper reports that swelling study in urea solutions and water, and sorption study of lauths violet by a novel type of new polymeric adsorbent containing AAm, AMPS and PEG. The aim of this study is to investigate the swelling properties and to increase the water absorption capacity of a series of new interpenetrating polymeric networks that are crosslinked a "new" multifunctional crosslinker such as TMPTA and using a hydrophilic ionic/anionic comonomer such as AMPS with a polymer such as PEG was selected. "New" AAm and AMPS based hydrogels and semi IPNs as potential polymeric adsorbents were prepared by free radical solution polymerization. It was of inter- 
est to swelling properties of AAm/AMPS hydrogels and AAm/AMPS/ PEG semi IPNs in urea solutions for new hydrogels synthesis for urea treatment as new membranes or crosslinked polymeric carriers, or dye sorption capacity of AAm/AMPS hydrogels and AAm/AMPS/ PEG semi IPNs hydrogels adsorption. Then, swelling properties and sorption properties of these hydrogel systems were studied.

\section{Experimental}

\subsection{Preparation of AAm/AMPS Hydrogels and AAm/AMPS/PEG Semi IPNs}

Highly swollen acrylamide (AAm) /2-acrylamido-2-met hyl-1-propanesulfonic acid (AMPS) hydrogels and semi-interpenetrating polymer networks, AAm/ AMPS/ PEG hydrogels with $0.25,0.50,0.75$ and $1.00 \mathrm{~g}$ poly (ethylene glycol) (PEG, $M_{w}=4600$ ), (Aldrich, Steinheim, Germany) (for per $1.00 \mathrm{~g} \mathrm{AAm}$ ) were prepared by free radical solution polymerization in aqueous solution of AAm monomer (Aldrich, Steinheim, Germany) with AMPS (Aldrich, Steinheim, Germany) as co-monomer and multifunctional crosslinker such as trimethylolpropane triacrylate (TMPTA) (Aldrich, Steinheim, Germany). For this study, the modes of specifications of the sources of water, the monomers, and other used chemicals were given in our previous study [6]. But, here, it will be mentioned to the preparation details and conditions of the hydrogels, again. The initiator, ammonium persulphate (APS) (Merck, Darmstadt, Germany) and the activator $N, N, N^{\prime}, \quad N$ '-tetramethylethylenediamine (TEMED) were also supplied by (Merck Schuchardt, Germany), and used as the redox initiator pair. All chemicals were used as received.

To prepare highly swollen AAm/AMPS hydrogel systems, AAm weighing $1.0 \mathrm{~g}(14.07 \mathrm{mmol})$ was dissolved in $1.0 \mathrm{~mL}$ water. Then $0 \mathrm{mg}, 60 \mathrm{mg} / 0.29 \mathrm{mmol}, 120 \mathrm{mg} /$ $0.58 \mathrm{mmol}, 180 \mathrm{mg} / 0.87 \mathrm{mmol}, 240 \mathrm{mg} / 1.16 \mathrm{mmol}, 300$ $\mathrm{mg} / 1.45 \mathrm{mmol}$ of AMPS were added to each AAm solutions, respectively. After these additions, for the synthesis, $0.25 \mathrm{~mL} / 0.0093 \mathrm{mmol}$ of $1 \%$ concentration of TMPTA and $0.20 \mathrm{~mL} / 0.044 \mathrm{mmol}$ aqueous solutions of APS (5.0 g APS $/ 0.022 \mathrm{~mol} / 100 \mathrm{~mL}$ water) and 0.25 $\mathrm{mL} / 0.017 \mathrm{mmol} 1 \%$ concentration of TEMED were added these aqueous solutions. The solutions were placed in PVC straws of $3 \mathrm{~mm}$ diameter, then, they were waited for gelation time in an oven at $40^{\circ} \mathrm{C}$. Fresh hydrogels obtained in long cylindrical shapes were cut into pieces of $3 \mathrm{~mm}-4 \mathrm{~mm}$ in length.

After gelation, they were washed and thoroughly rinsed with distilled water, blot dried with filter paper, dried in air and vacuum, and stored for swelling and sorption studies.

\subsection{Measurement of Swelling in Water and Aqueous Urea}

For swelling studies, AAm/AMPS hydrogels and AAm/ AMPS/PEG semi IPNs were accurately weighted and transferred into water or aqueous $0.01 \mathrm{M} / 0.03 \mathrm{M}$ urea solutions. Urea was provided from Merck, Darmstadt, Germany. Dry gels were weighed and then immersed in distilled water, or aqueous $0.01 \mathrm{M}$ or $0.03 \mathrm{M}$ urea solutions at $25 \pm 0.1^{\circ} \mathrm{C}$. Swollen gels were removed from water or aqueous solutions at predetermined times, blotted dry, and weighed in air. The measurements were conducted at $25 \pm 0.1^{\circ} \mathrm{C}$ in a water bath.

\subsection{Dye Sorption Equilibrium Experimental}

Batch sorption studies were applied in all sorption experiments. Cationic dye, Lauths violet, (Thionin, LV), used in sorption studies and some properties of LV were given in Table 1. Cationic dye, Lauths violet was purchased from Aldrich, Milwauke, USA.

Solutions of LV concentration range $1.00 \times 10^{-6} \mathrm{M}$ $20.0 \times 10^{-6} \mathrm{M}$ in distilled water were prepared. Aam /AMPS hydrogels and AAm/AMPS/PEG semi IPNs containing $180 \mathrm{mg}$ AMPS was used in a known volume of dye solution until equilibrium was reached. For AMPS effect on the dye sorption, aqueous solution of concentration of $20.0 \times 10^{-6} \mathrm{M}$ of LV was used.

After sorption, dye solution was separated by decantation from the hydrogels. Spectrophotometric method was applied to dye solutions for determining of the concentrations of the hydrogel systems. Spectrophotometric measurements were carried out using a SHIMADZU UV 1601 model UV-VIS spectrophotometer at ambient temperature. The absorbances of these solutions were read at $598 \mathrm{~nm}$ for LV [32]. Distilled water was chosen as the reference. The equilibrium concentrations of the cationic dye solutions were determined by means of precalibrated scales.

\section{Results and Discussion}

Semi-interpenetrating network (semi IPNs) hydrogel, composed of acrylamide (AAm) with 2-acrylamido-2methyl-1-propanesulfonic acid (AMPS) as co-monomer, with poly (ethylene glycol) (PEG) and a multifunctional crosslinker such as trimethylolpropane triacrylate (TMPTA) was prepared. AAm/AMPS hydrogels and AAm/AMPS/PEG semi IPNs were synthesized by free radical solution polymerization (Figure 1). 
Table 1. Some properties of lauths violet.

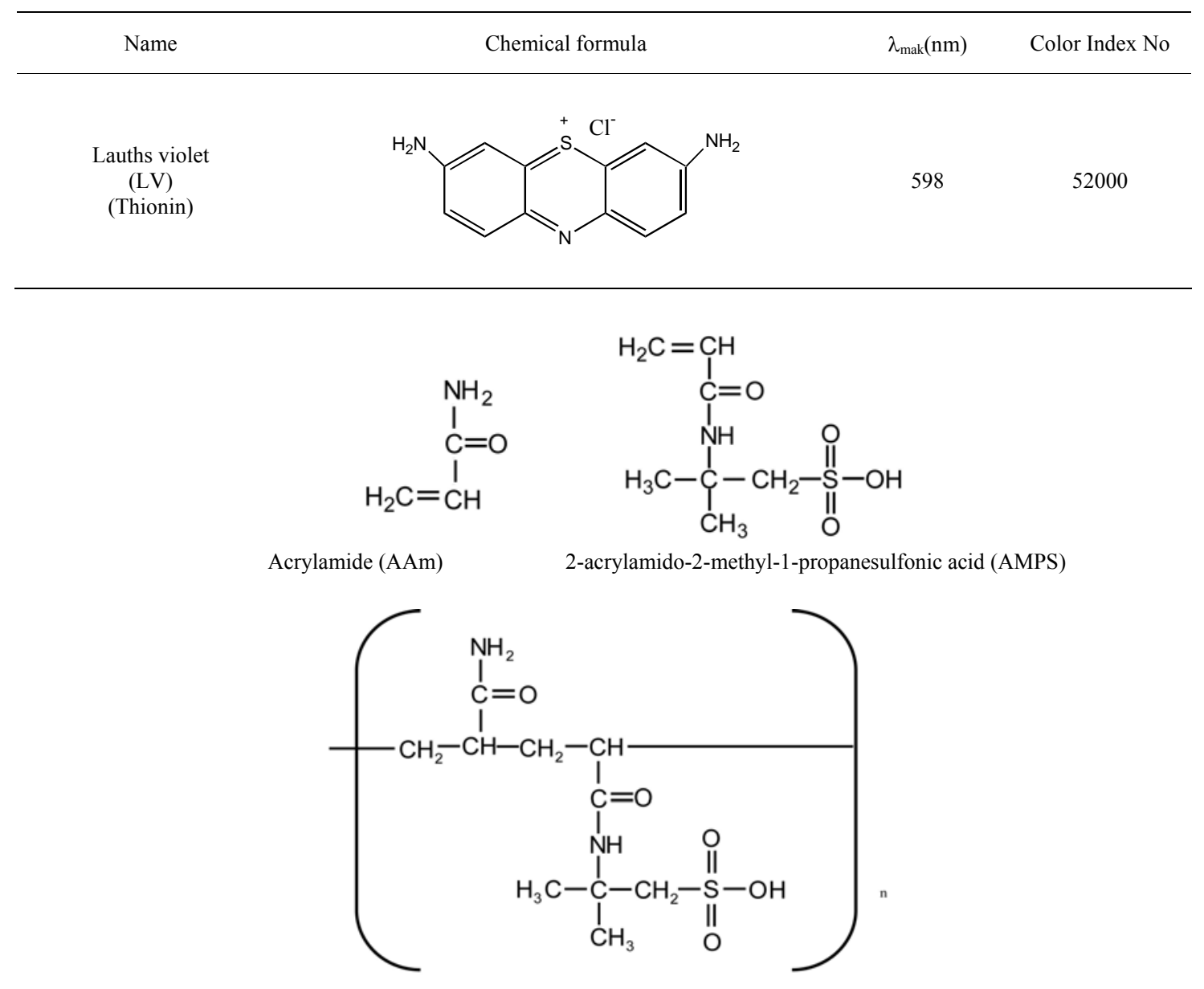

Acrylamide/2-acrylamido-2-methyl-1-propanesulfonic acid (AAm/AMPS)

Figure 1. Representative chemical structures of monomers and AAm/AMPS hydrogel.

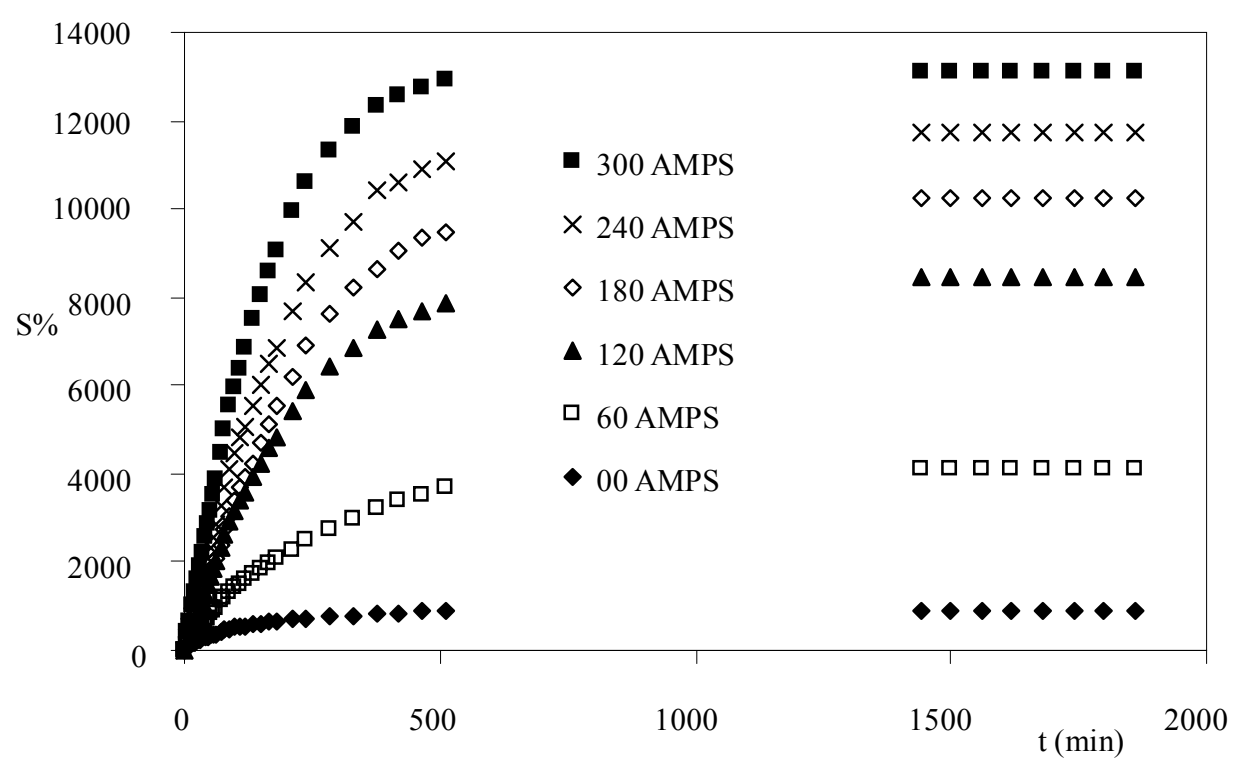

Figure 2. Swelling isotherms of AAm/AMPS hydrogels crosslinked in aqueous $0.01 \mathrm{M}$ urea solutions. 


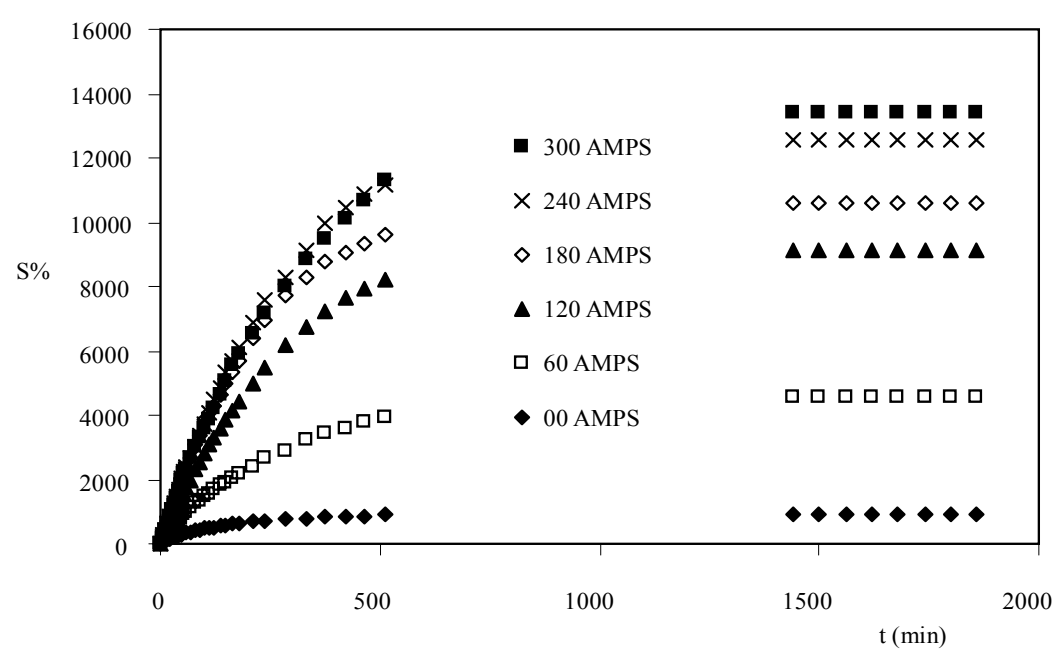

Figure 3. Swelling isotherms of AAm/AMPS hydrogels crosslinked in aqueous $0.03 \mathrm{M}$ urea solutions.

\subsection{Equilibrium Swelling Studies}

Swelling experiments were performed in water at $25^{\circ} \mathrm{C}$, gravimetrically. Some swelling and diffusion parameters of AAm/AMPS hydrogels and AAm/AMPS/PEG semi IPNs in water were reported previously by our group [6].

A fundamental relationship exists between the swelling of a polymer in a solvent and the nature of the polymer and the solvent. The percentage swelling ( $\mathrm{S} \%$ ) of the hydrogels in distilled water was calculated from the following relation,

$$
S \%=\frac{m_{t}-m_{o}}{m_{o}} \times 100
$$

where $m$ is the mass of the swollen gel at time $t$ and $m_{0}$ is the mass of the dry gel at time 0 .

The water intake of initially dry hydrogels was followed for AAm/AMPS hydrogels and AAm/AMPS/PEG semi IPNs crosslinked by TMPTA in water, and swelling isotherms of AAm/AMPS hydrogels and AAm/AMPS/ PEG semi IPNs crosslinked by TMPTA were presented in our published study [6].

Swelling studies have been repeated in aqueous 0.01 $\mathrm{M}$ and $0.03 \mathrm{M}$ urea solutions for AAm/AMPS hydrogels and AAm/AMPS/PEG semi IPNs crosslinked by TMPTA. Swelling isotherms of AAm/AMPS hydrogels in aqueous $0.01 \mathrm{M}$ urea solutions was presented at Figure 2, and swelling isotherms of AAm/AMPS hydrogels in aqueous $0.03 \mathrm{M}$ urea solutions at Figure 3. Figure 2 and Figure 3 show that swelling increases with time up to certain level, then levels off. This value of swelling may be called as the equilibrium percent swelling $\left(\mathrm{S}_{\mathrm{eq}} \%\right)$. $\mathrm{S}_{\mathrm{eq}} \%$ of AAm/AMPS hydrogels and AAm/AMPS/PEG semi IPNs is used for the calculation of swelling characterization parameters. $\mathrm{S}_{\mathrm{eq}} \%$ AAm/AMPS hydrogels and AAm/AMPS/PEG semi IPNs are given in Table 2.
Table 2 shows that the values of $\mathrm{S}_{\mathrm{eq}} \%$ of AAm hydrogels are $900 \%$, but $\mathrm{S}_{\mathrm{eq}} \%$ of AAm/AMPS hydrogels are $4850 \%-12750 \%$ with the incorporation of AMPS groups into AAm chains crosslinked by TMPTA for water. Table 2 shows that the values of $S_{\text {eq }} \%$ of AAm hydrogels are 900 and $950 \%$, but $\mathrm{S}_{\mathrm{eq}} \%$ of AAm/AMPS hydrogels are $4100 \%-13450 \%$ with the incorporation of AMPS groups into AAm chains crosslinked by TMPTA in aqueous $0.01 \mathrm{M}$ and $0.03 \mathrm{M}$ ures solutions.

In Table 2, $\mathrm{S}_{\mathrm{eq}} \%$ of the hydrogels increased with the AMPS content in the copolymers. $S_{\text {eq }} \%$ of AAm/AMPS hydrogels is higher than $\mathrm{S}_{\mathrm{eq}} \%$ of AAm hydrogels. The reason of this is the hydrophilic groups on the AMPS. AMPS has got strongly ionizable sulfonate group. The more hydrophilic groups in the AAm/AMPS get the more the swelling of the AAm/AMPS hydrogels. This is an expected result about swelling of AAm/AMPS hydrogel systems. Hydrophilicity of AAm/AMPS copolymers becomes greater than that of AAm, so, the swelling of AAm/AMPS copolymers is greater than the swelling of AAm hydrogels [6].

Effect of the content of AMPS and concentration of aqueous urea solutions onto swelling of AAm/AMPS hydrogels crosslinked by TMPTA in water, in aqueous $0.01 \mathrm{M}$ urea solutions and in aqueous $0.03 \mathrm{M}$ urea solutions are presented at Figure 4.

The values of $\mathrm{S}_{\mathrm{eq}} \%$ of AAm/AMPS hydrogels swollen in water are generally smaller than the hydrogels swollen in urea solutions. The reason of this different behavior is the hydrophilic character of urea molecules. Urea molecule has got more hydrophilic sites, as $\mathrm{NH}_{2}$ and $\mathrm{C}=\mathrm{O}$. When, urea molecules have interacted with much water, so, there has been much swelling than swelling values in water, also. That's way, urea molecules have got hydrophilic groups, more swelling values have been observed when the hydrogels swollen in aqueous urea solutions. 
Table 2. Values of the equilibrium percentage swelling $\left(S_{\mathrm{eq}} \%\right)$ and equilibrium water contents (EWC) or equilibrium urea/water contents (EUWC) of AAm/AMPS hydrogels and AAm/AMPS/PEG (containing 0.50 g PEG) semi IPNs systems.

\begin{tabular}{|c|c|c|c|}
\hline & water & $0.01 \mathrm{M}$ urea & $0.03 \mathrm{M}$ urea \\
\hline AMPS / mg & \multicolumn{3}{|c|}{ Equilibrium percentage swelling, $\left(\mathrm{S}_{\mathrm{eq}} \%\right)$} \\
\hline 0 & 900 & 900 & 950 \\
\hline 60 & 4850 & 4100 & 4500 \\
\hline 120 & 7250 & 8500 & 9100 \\
\hline 180 & 10400 & 10300 & 10600 \\
\hline 240 & 11150 & 11750 & 12550 \\
\hline 300 & 12750 & 13100 & 13450 \\
\hline \multicolumn{4}{|l|}{ AMPS/PEG mg } \\
\hline 0 & 550 & 500 & 500 \\
\hline 60 & 1100 & 1350 & 1350 \\
\hline 120 & 2100 & 2100 & 2050 \\
\hline 180 & 2650 & 3250 & 3250 \\
\hline 240 & 2950 & 3400 & 3400 \\
\hline 300 & 4400 & 3600 & 3650 \\
\hline $\mathrm{AMPS} / \mathrm{mg}$ & \multicolumn{3}{|c|}{ Equilibrium water content, (EWC)/Equilibrium urea/water content, (EUWC) } \\
\hline 0 & 0.8995 & 0.9011 & 0.9042 \\
\hline 60 & 0.9798 & 0.9763 & 0.9784 \\
\hline 120 & 0.9864 & 0.9883 & 0.9891 \\
\hline 180 & 0.9905 & 0.9904 & 0.9906 \\
\hline 240 & 0.9911 & 0.9915 & 0.9921 \\
\hline 300 & 0.9922 & 0.9924 & 0.9926 \\
\hline \multicolumn{4}{|l|}{ AMPS/PEG mg } \\
\hline 0 & 0.8422 & 0.8366 & 0.8385 \\
\hline 60 & 0.9163 & 0.9315 & 0.9317 \\
\hline 120 & 0.9542 & 0.9541 & 0.9532 \\
\hline 180 & 0.9639 & 0.9703 & 0.9700 \\
\hline 240 & 0.9674 & 0.9832 & 0.9716 \\
\hline 300 & 0.9777 & 0.9947 & 0.9734 \\
\hline
\end{tabular}

Here main characteristic effects are the hydrophilic character of urea molecules. So, the more hydrophilic groups in the aqueous urea solutions get the more the swelling of AAm/AMPS hydrogels and AAm/AMPS/PEG semi IPNs.

\subsection{Equilibrium Water or Urea/Water Content}

The water (or with together urea,) absorbed by AAm /AMPS hydrogels and AAm/AMPS/PEG semi IPNs is quantitatively represented by Equilibrium water content
(EWC), or Equilibrium urea/water content (EUWC), by using below equation $[33,34]$

$$
\mathrm{EWC}=\frac{m_{s}-m_{o}}{m_{s}}
$$

Here, $m_{s}$ is the mass of the swollen gel at time $t$ (equilibrium), and $\mathrm{m}_{\mathrm{o}}$ is the mass of the dry gel at time 0 . The EWC (or EUWC) values of AAm/AMPS hydrogels and AAm/AMPS/PEG semi IPNs were calculated. The EWC (or EUWC) values of the hydrogels are tabulated in Table 2. Generally, it was seen that the EWC (or 


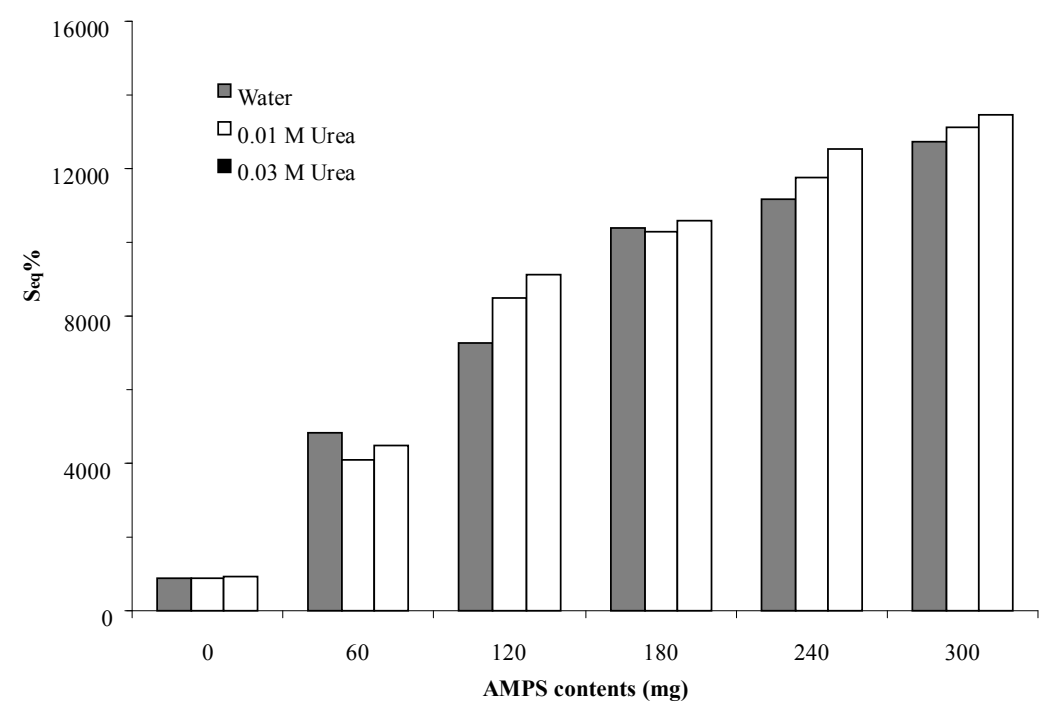

Figure 4. Effect of the content of AMPS onto swelling of AAm/AMPS hydrogels in water, in aqueous $0.01 \mathrm{M}$ urea solutions and in aqueous $0.03 \mathrm{M}$ urea solutions.

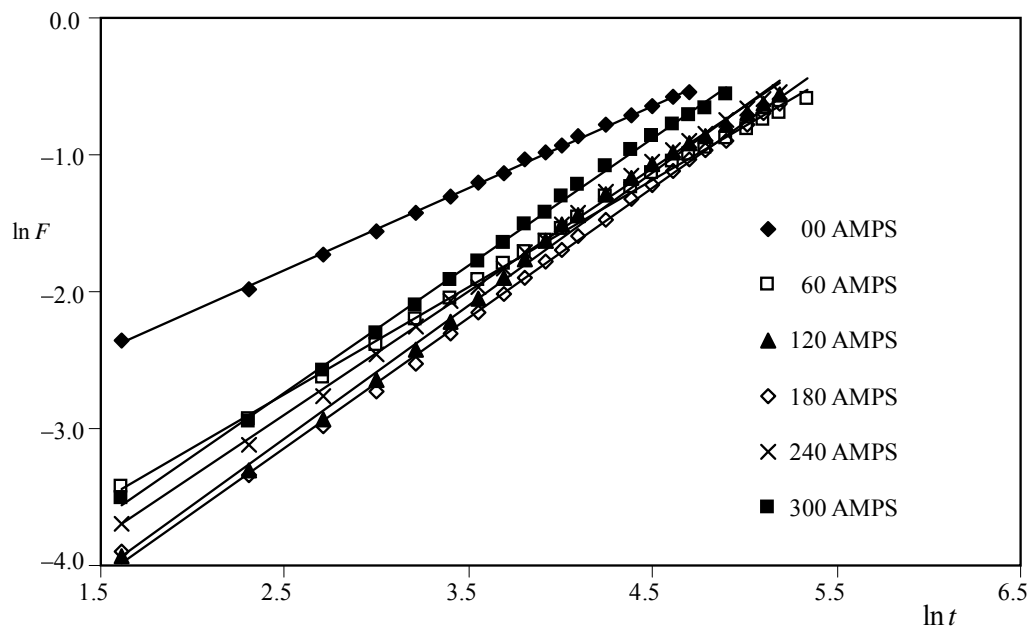

Figure 5. Plots of InF versus Int for AAm/AMPS hydrogels in aqueous $0.01 \mathrm{M}$ urea solutions.

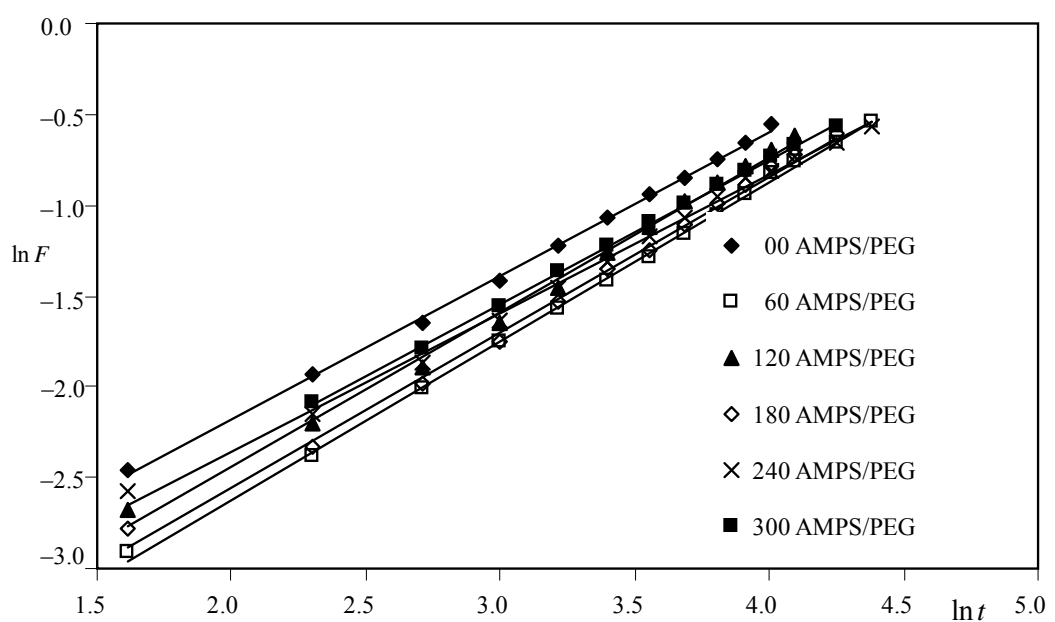

Figure 6. Plots of InF versus Int for AAm/AMPS/PEG hydrogels in aqueous 0.01 M urea solutions. 
EUWC) values of the hydrogels are increased by the adding of AMPS molecules. Here, the main effect is the hydrophilic character of AMPS.

It is well known that the swelling of a hydrogel is induced by electrostatic repulsion of the ionic charges of its network. The ionic charge content is important. AMPS contain many strongly ionizable sulfonate group. The swelling increase is due to an increase in the anionic units. The hydrophilic group numbers of Aam /AMPS hydrogels and AAm/AMPS/PEG semi IPNs are higher than those of AAm, and so the swelling values of AAm/ AMPS hydrogels and AAm/AMPS/PEG semi IPNs are greater than that of AAm swelling values.

\subsection{Diffusion}

When a glassy hydrogel is brought into contact with water, water diffuses into the hydrogel and the network expands resulting in swelling of the hydrogel. Diffusion involves migration of water into pre-existing or dynamically formed spaces between hydrogel chains. Swelling of the hydrogel involves larger segmental motion resulting, ultimately, in increased separation between hydrogel chains.

Analysis of the mechanisms of water diffusion into swellable polymeric systems has received considerable attention in recent years, because of important applications of swellable polymers in biomedical, pharmaceutical, environmental, and agricultural engineering.

The following equation is used to determine the nature of diffusion of water into hydrogels [33,35].

$$
F=\frac{M_{t}}{M_{s}}=k t^{n}
$$

Here, $F$ is the fractional uptake at time $t, M_{t}$ and $M_{s}$ are the mass uptake of the solvent at time $t$ and the equilibrium, respectively. Eq. 3 is valid for the first $60 \%$ of the fractional uptake. Fickian diffusion and Case II transport are defined by $n$ values of 0.5 and 1.0 , respectively. Anomalous transport behavior (non-Fickian diffusion) is intermediate between Fickian and Case II. That is reflected by $\mathrm{n}$ between 0.5 and $1.0[33,35]$. The values of $(n)$ and $(k)$ were calculated from the slope and the intercept of the plot of $\ln F$ against lnt, respectively.

For AAm/AMPS hydrogels and AAm/AMPS/PEG semi IPNs, $\operatorname{lnF}$ vs. Int graphs are plotted and representative results are shown in Figure 5 and Figure 6. Diffusional exponents, (n) and diffusion constant, (k) are calculated and listed in Table 3.

Table 3 shows that the number determining the type of diffusion (n) is over 0.50 . Hence the diffusion of water into the super water-retainer hydrogels is generally found to have a non-Fickian character. When the diffusion type is anomalous behavior, the relaxation and diffusion time are of the same order of magnitude. As solvent diffuses into the hydrogel, rearrangement of chains does not occur immediately.

The study of diffusion phenomena of water in hydrogels is of value in that it clarifies polymer behavior. For hydrogel characterization, the diffusion coefficients can be calculated by various methods. The diffusion coefficient, D of the water was calculated using the following equation $[33,36,37]$.

$$
D=\pi r^{2}\left(\frac{k}{4}\right)^{1 / n}
$$

Here, $\mathrm{D}$ is in $\mathrm{cm}^{2} \mathrm{~min}^{-1}, \mathrm{r}$ is the radius of a cylindrical polymer sample, $(\mathrm{n})$ is the diffusional exponent and $(\mathrm{k})$ is a constant incorporating characteristic of the macromolecular network system and the penetrant. The values of diffusion coefficient determined for AAm/AMPS hydrogels and AAm/AMPS/PEG semi IPNs are listed in Table 3. Table 3 shows that the values of the diffusion coefficient of AAm/AMPS hydrogels and AAm/AMPS /PEG semi IPNs vary from $0.11 \times 10^{-3} \mathrm{~cm}^{2} \mathrm{~min}^{-1}$ to $112.53 \times 10^{-3} \mathrm{~cm}^{2} \mathrm{~min}^{-1}$. It was seen that an increasing of the values of the diffusion coefficient of Aam /AMPS hydrogels and AAm/AMPS/PEG semi IPNs by increasing of AMPS content. Hydrophilicity of AAm/AMPS hydrogels and AAm/AMPS/PEG semi IPNs becomes greater than that of AAm, so, the diffusion of water of AAm/AMPS hydrogels and AAm/AMPS/PEG semi IPNs is greater than the diffusion of water of AAm hydrogels.

\subsection{PEG Effect on the Swelling and Diffusion}

For investigation of the effect of mass/content of PEG on the swelling properties of AAm/AMPS/PEG semi IPNs, the related swelling isotherms of AAm/AMPS /PEG hydrogels were constructed and representative swelling isotherms, and $\operatorname{lnF}$ vs. lnt graphs are plotted and representative results are shown in Figure 7, Figure 8 and Figure 9. PEG effect on some swelling and diffusion parameters of AAm/AMPS/PEG semi IPN systems containing $180 \mathrm{mg}$ AMPS are tabulated in Table 4 .

It was shown that a decreasing of the equilibrium swelling percent $\left(\mathrm{S}_{\mathrm{eq}} \%\right)$, and equilibrium water contents (EWC/EUWC) of AAm/AMPS/PEG semi IPN systems when PEG has been added to the hydrogel systems. Incorporation of PEG into the copolymer network leads to lower degrees of swelling. It was seen that diffusion of water or urea/water mixture onto AAm/AMPS/PEG semi IPN systems has shown "non-Fickian" character from Table 4. Value of diffusion exponent of Aam 
Table 3. Some diffusion parameters of AAm/AMPS hydrogels and AAm/AMPS/PEG (containing 0.50 g PEG) semi IPNs systems.

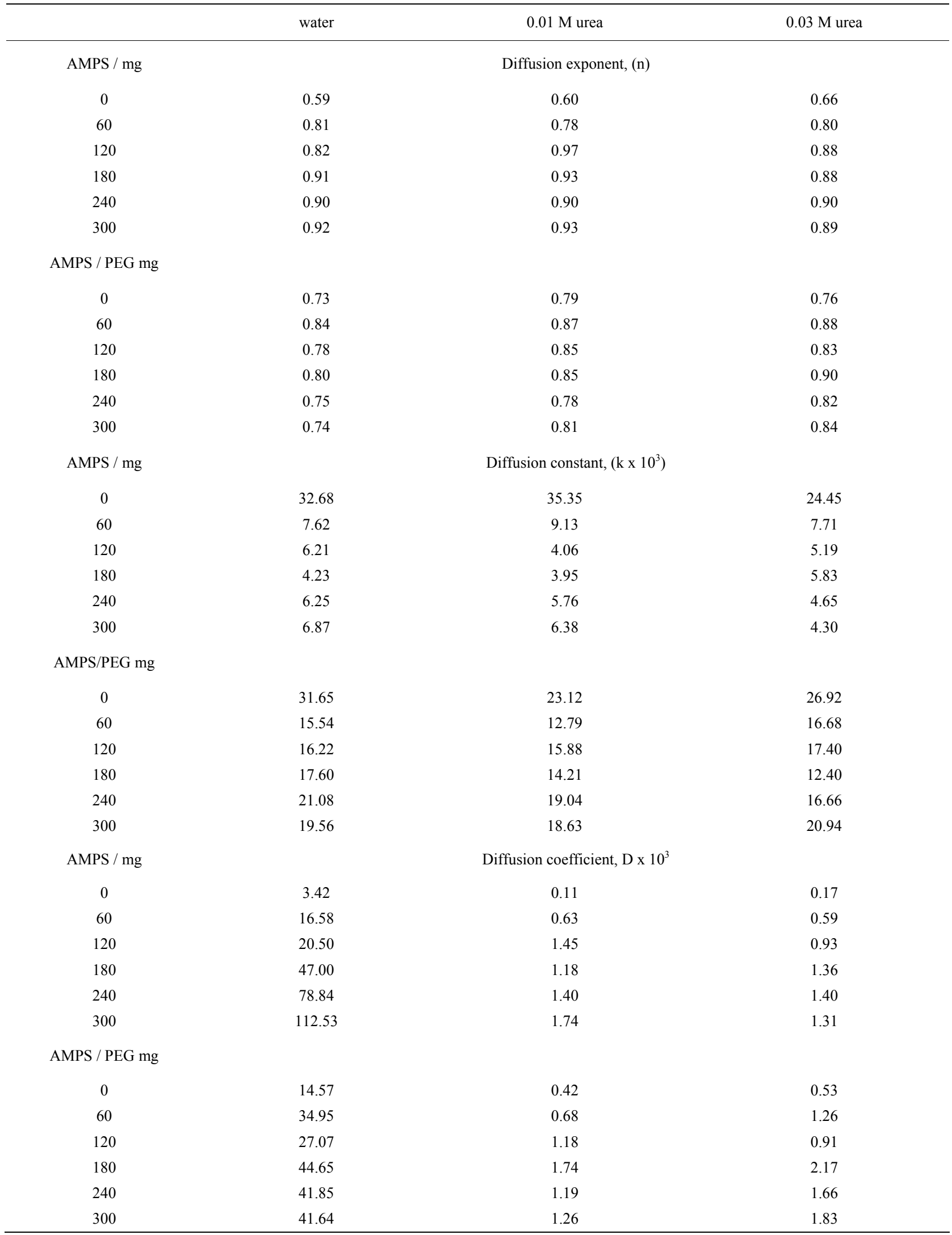




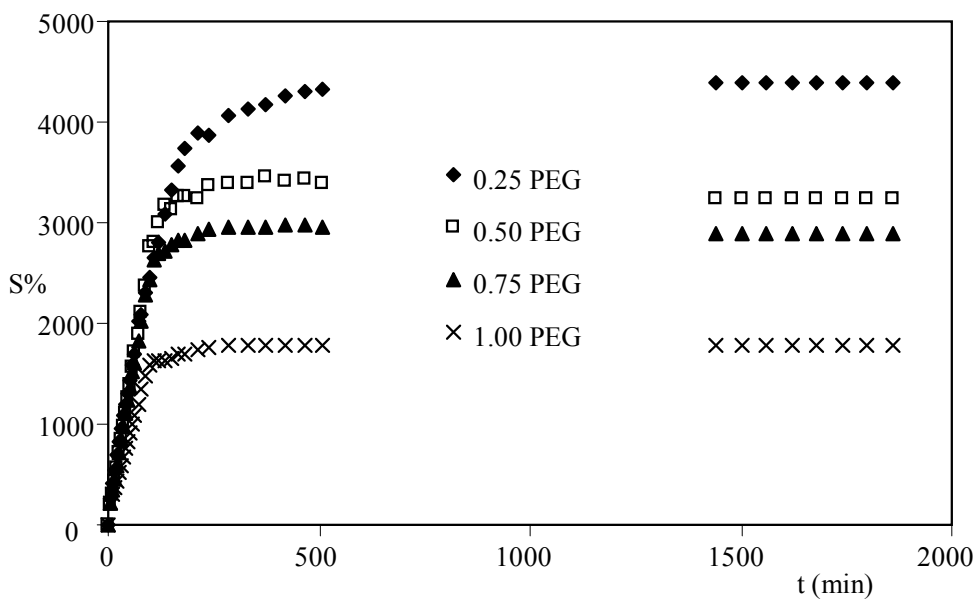

Figure 7. Effect of the content of PEG in swelling isotherms for AAm/AMPS/PEG hydrogels in aqueous $0.03 \mathrm{M}$ urea solutions.

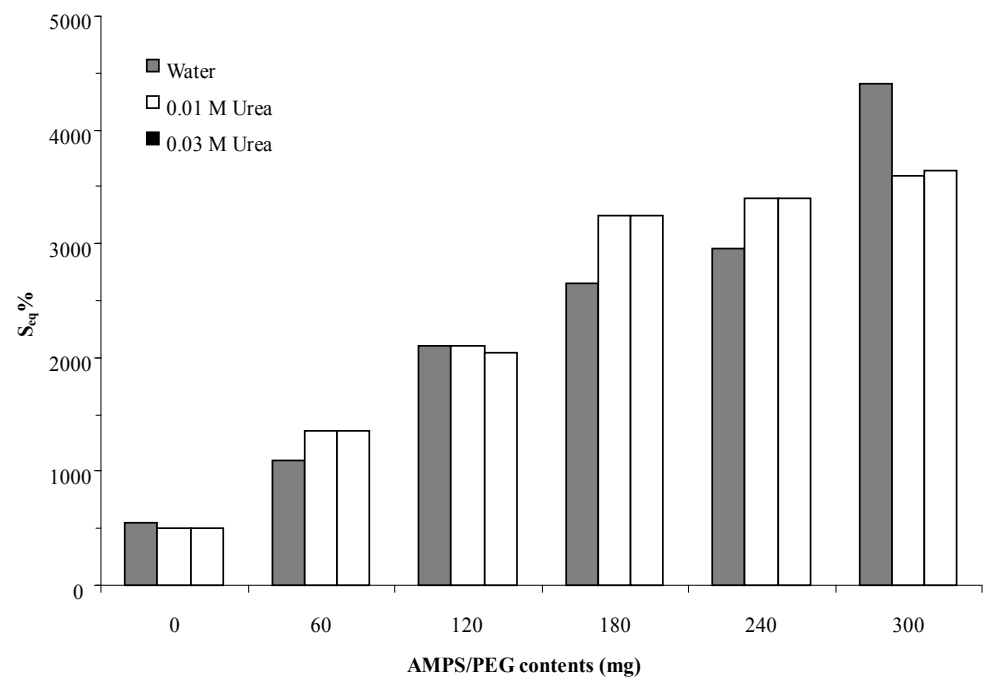

Figure 8. Effect of the content of AMPS onto swelling of AAm/AMPS/PEG hydrogels in water, in aqueous $0.01 \mathrm{M}$ urea solutions and in aqueous $0.03 \mathrm{M}$ urea solutions.

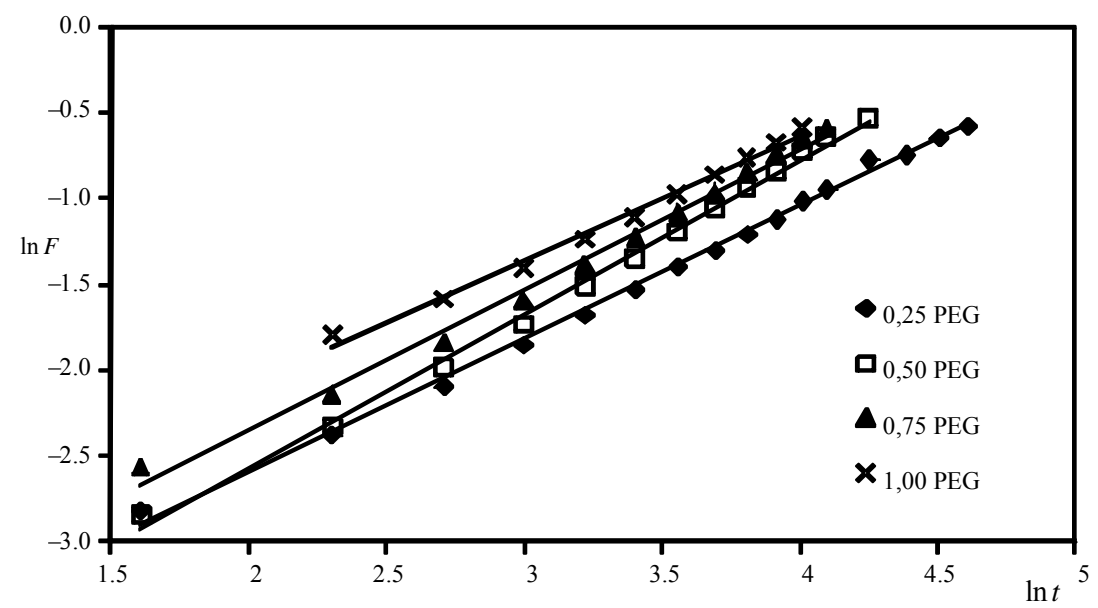

Figure 9. Effect of the content of PEG in swelling kinetics curves for AAm/AMPS/PEG hydrogels in aqueous $0.03 \mathrm{M}$ urea solutions. 
Table 4. Some swelling and diffusion parameters of AAm/AMPS/PEG semi IPNs systems containing 180 mg AMPS.

\begin{tabular}{|c|c|c|c|c|}
\hline PEG/g & 0.25 & 0.50 & 0.75 & 1.00 \\
\hline \multicolumn{5}{|c|}{ Equilibrium percentage swelling, $\left(\mathrm{S}_{\mathrm{eq}} \%\right)$} \\
\hline water & 3150 & 2650 & 2000 & 1500 \\
\hline $0.01 \mathrm{M}$ urea & 4350 & 3250 & 2250 & 1750 \\
\hline \multirow[t]{2}{*}{$0.03 \mathrm{M}$ urea } & 4400 & 3250 & 2900 & 1800 \\
\hline & \multicolumn{4}{|c|}{ Equilibrium water content, (EWC)/Equilibrium urea-water content, (EUWC) } \\
\hline water & 0.9694 & 0.9639 & 0.9527 & 0.9370 \\
\hline $0.01 \mathrm{M}$ urea & 0.9774 & 0.9703 & 0.9570 & 0.9432 \\
\hline \multirow[t]{2}{*}{$0.03 \mathrm{M}$ urea } & 0.9777 & 0.9700 & 0.9666 & 0.9469 \\
\hline & \multicolumn{4}{|c|}{ Diffusion exponent, $\mathrm{n}$} \\
\hline water & 0.74 & 0.80 & 0.70 & 0.81 \\
\hline $0.01 \mathrm{M}$ urea & 0.74 & 0.85 & 0.77 & 0.75 \\
\hline \multirow[t]{2}{*}{$0.03 \mathrm{M}$ urea } & 0.79 & 0.90 & 0.83 & 0.76 \\
\hline & \multicolumn{4}{|c|}{ Diffusion constant, $\mathrm{k} \times 10^{3}$} \\
\hline water & 19.56 & 17.60 & 29.77 & 25.09 \\
\hline $0.01 \mathrm{M}$ urea & 16.59 & 14.21 & 20.06 & 19.73 \\
\hline \multirow[t]{2}{*}{$0.03 \mathrm{M}$ urea } & 14.96 & 12.40 & 18.21 & 25.46 \\
\hline & \multicolumn{4}{|c|}{ Diffusion coefficient, $\mathrm{D} \times 10^{3}$} \\
\hline water & 34.44 & 44.65 & 29.79 & 48.96 \\
\hline $0.01 \mathrm{M}$ urea & 0.88 & 1.74 & 0.52 & 0.66 \\
\hline $0.03 \mathrm{M}$ urea & 1.12 & 2.16 & 1.71 & 1.02 \\
\hline
\end{tabular}

Table 5. Some adsorption parameters of AAm/AMPS hydrogels and AAm/AMPS/PEG (containing 0.5 g PEG) semi -IPNs systems in aqueous solutions of $20.0 \times 10^{-6} \mathrm{M}$ LV solutions.

\begin{tabular}{|c|c|c|c|c|c|}
\hline AMPS, mg & 60 & 120 & 180 & 240 & 300 \\
\hline & \multicolumn{5}{|c|}{ Dye removal capacity, $\mathrm{q} \times 10^{5}\left(\mathrm{~mol} \mathrm{~g}^{-1}\right)$} \\
\hline & 1.25 & 1.23 & 1.35 & 1.28 & 1.06 \\
\hline & \multicolumn{5}{|c|}{ Adsorption percentage, Ads $\%$} \\
\hline & 71.75 & 82.79 & 89.01 & 90.09 & 92.66 \\
\hline & \multicolumn{5}{|c|}{ Partition coefficient, $K_{d}$} \\
\hline & 2.54 & 4.81 & 8.10 & 9.09 & 12.63 \\
\hline \multirow[t]{7}{*}{ AMPS/PEG, mg } & 60 & 120 & 180 & 240 & 300 \\
\hline & \multicolumn{5}{|c|}{ Dye removal capacity, $\mathrm{q} \times 10^{5}\left(\mathrm{~mol} \mathrm{~g}^{-1}\right)$} \\
\hline & 1.12 & 1.23 & 0.94 & 1.30 & 1.22 \\
\hline & \multicolumn{5}{|c|}{ Adsorption percentage, Ads $\%$} \\
\hline & 88.27 & 92.02 & 93.20 & 96.09 & 96.31 \\
\hline & \multicolumn{5}{|c|}{ Partition coefficient, $\mathrm{K}_{\mathrm{d}}$} \\
\hline & 7.52 & 11.53 & 13.70 & 24.61 & 26.10 \\
\hline
\end{tabular}

Table 6. PEG affects on some adsorption parameters of AAm/AMPS/PEG semi IPNs systems crosslinked by TMPTA containing $180 \mathrm{mg}$ AMPS in aqueous solutions of $20.0 \times 10^{-6} \mathrm{M} \mathrm{LV}$ solutions.

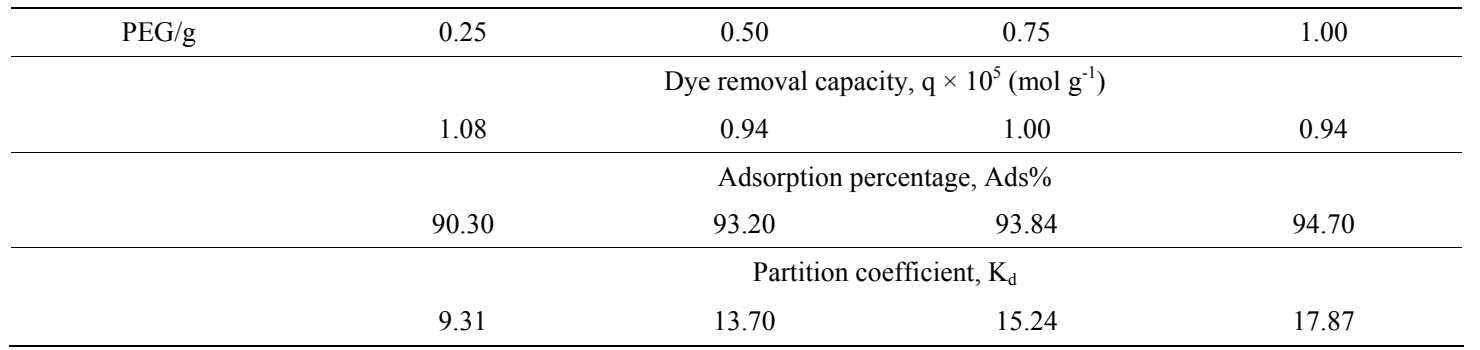


/AMPS/PEG semi IPN systems are between $0.70-0.90$. On the other hand, also, it is seen that an increasing of diffusion constant $(\mathrm{k})$ of AAm/AMPS/PEG hydrogels with increasing content of PEG in hydrogel systems from Table 4. Here, it was said that PEG chains was placed in the crosslinked polymeric systems, in stead of crosslinked AAm and AMPS molecules, it was seen that decreasing of the value of the equilibrium swelling percent and related parameters, because of decreasing of hydrophilic character at crosslinked polymeric systems. In addition of this phenomenon, the PEG chains are located in the free space of crosslinked polymer networks; water diffusion is prevented by the PEG chains. This is also caused of decreasing of the equilibrium swelling percent and related parameters.

\subsection{Equilibrium Sorption Studies}

For sorption of cationic dye, Lauths violet (LV) into the hydrogels, AAm/AMPS hydrogels and AAm/AMPS/PEG semi IPNs were placed in aqueous LV solutions and allowed to equilibrate for four days at $25^{\circ} \mathrm{C}$. At the end of this period AAm/AMPS hydrogels and AAm/AMPS /PEG semi IPNs in aqueous LV solutions showed the dark coloration. But acrylamide hydrogel did not sorb any dye from solution.

For equilibrium sorption studies, the dye removal capacity (q) (mass amount as "mol" of sorption per unit mass (as gram)) of the adsorbent, adsorption percentage (Ads\%), and partition coefficient $\left(\mathrm{K}_{\mathrm{d}}\right)$ can be investigated.

The dye removal capacity, (q) of AAm/AMPS hydrogels and AAm/AMPS/PEG semi IPNs were evaluated by using the following equation.

$$
q=\frac{\left(C_{o}-C\right) v}{m}
$$

Where $\mathrm{q}$, is the dye removal capacity of AAm/AMPS hydrogels and AAm/AMPS/PEG semi IPNs (mol g ${ }^{-1}$ ), $\mathrm{Co}$ and $\mathrm{C}$ are the concentration of aqueous LV solutions in the initial solution and the aqueous phase after treatment for a certain period time, respectively $\left(\mathrm{mol} \mathrm{L}^{-1}\right), \mathrm{v}$ is the volume of the aqueous phase (L) and $\mathrm{m}$ is the amount of dry AAm/AMPS hydrogels and AAm /AMPS/PEG semi IPNs (g).

Uptake of dye was measured effects of contents of AMPS. The dye removal capacity, the amount of dyes sorbed onto unit dry mass of the gel was calculated for uptake of dye within the hydrogel in $20.0 \times 10^{-6} \mathrm{~mol} \mathrm{LV}$ in $\mathrm{L}$ of aqueous solutions, and presented in Table 5. Table 5 presents that the dye removal capacity of Aam /AMPS hydrogels and AAm/AMPS/PEG semi IPNs $\left(0.94 \times 10^{-5}-1.35 \times 10^{-5} \mathrm{~mol} \mathrm{~g}^{-1}\right)$.
Equilibrium LV adsorption isotherm of AAm/AMPS hydrogels and AAm/AMPS/PEG semi IPNs is presented in Figure 10 and Figure 11. To Figure 10 and Figure 11, the dye removal capacity (mol amount of sorption LV per unit mass) of the hydrogels is increased with the increasing concentration LV sorbed onto unit dry mass of the gel, q of the hydrogels increased with PEG in the copolymers. The reason of this is the hydrophilic effect and dye sorption capability of PEG.

Adsorption percentage (Ads\%) of AAm/AMPS hydrogels and AAm/AMPS/PEG semi IPNs was calculated by following equation.

$$
A d s \%=\frac{C_{o}-C}{C_{o}} \times 100
$$

Here Co and $\mathrm{C}$ were defined earlier.

Table 5 presents that adsorption percentage of them $(71.75 \%-96.31 \%)$ is increased with increased with the increasing of content of AMPS. The dye removal capacity and adsorption percentage of AAm/AMPS hydrogels and AAm/AMPS/PEG semi IPNs gradually increased with the increase of content of AMPS in hydrogels and semi IPNs.

Partitioning of dissolved constituents between an aqueous phase and adsorbents in waters and sediments has commonly been described by an empirical partition coefficient that simply relates the total concentration of a dissolved species to the total concentration of the adsorbed species [38,39].

$$
K_{d}=\frac{\left(C_{o}-C\right)}{C}
$$

Here; $\mathrm{Kd}$ is empirical partition coefficient at equilibrium. Co and $\mathrm{C}$ were defined earlier. Partition coefficients of LV between dye solution and hydrogels were calculated, and are shown in Table 5. In Table 5, $\mathrm{Kd}$ values of AAm/AMPS hydrogels is $2.54-12.63$, but $\mathrm{Kd}$ values of AAm/AMPS/PEG semi IPNs is $7.52-26.10$ with the incorporation of PEG groups into the hydrogels. Here, Kd values of AAm/AMPS hydrogels and Aam /AMPS/PEG semi IPNs are higher than 1.0. So, it can be said that synthesized crosslinked AAm/AMPS hydrogels and AAm/AMPS/PEG semi IPNs could be used as potential water adsorbent [38,39].

The ionic charge content in the polymeric structure is important. AMPS contain ionic units. The swelling degrees of the hydrogels increase due to increasing of the hydrophilic units on hydrogel structure (Figure 1). Therefore AAm/AMPS hydrogels and AAm/AMPS /PEG semi IPNs have many ionic groups that can increase interaction between the cationic dye molecules and anionic groups of hydrogels. The results of swelling studies are parallel character to the results of sorption 


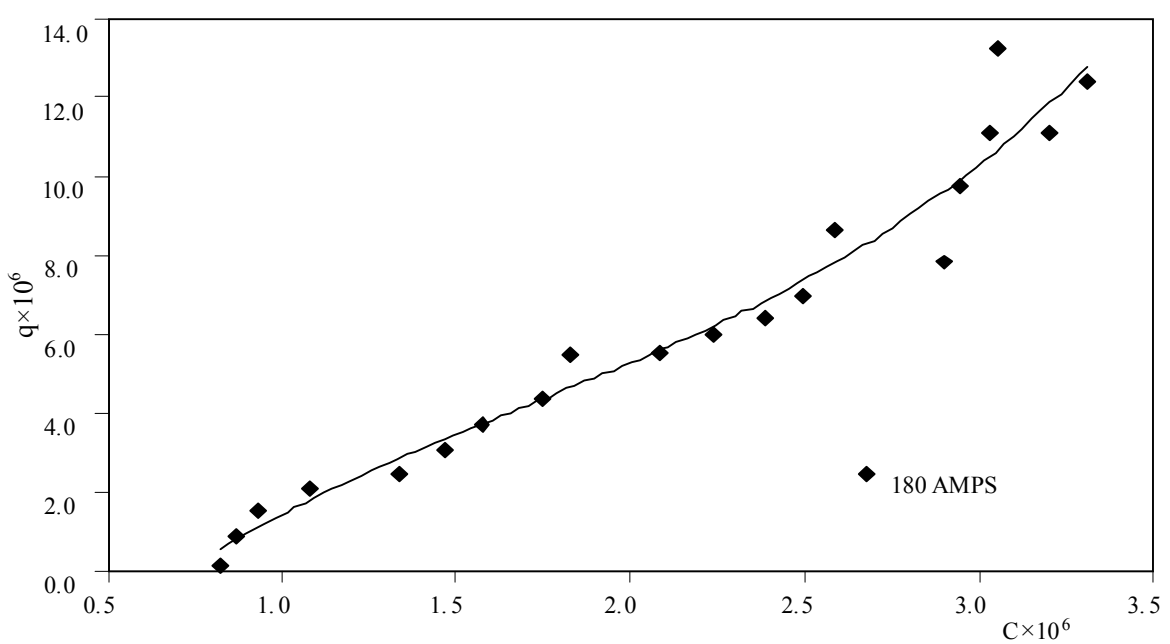

Figure 10. Equilibrium sorption isotherms of AAm/AMPS hydrogels in aqueous LV solutions.

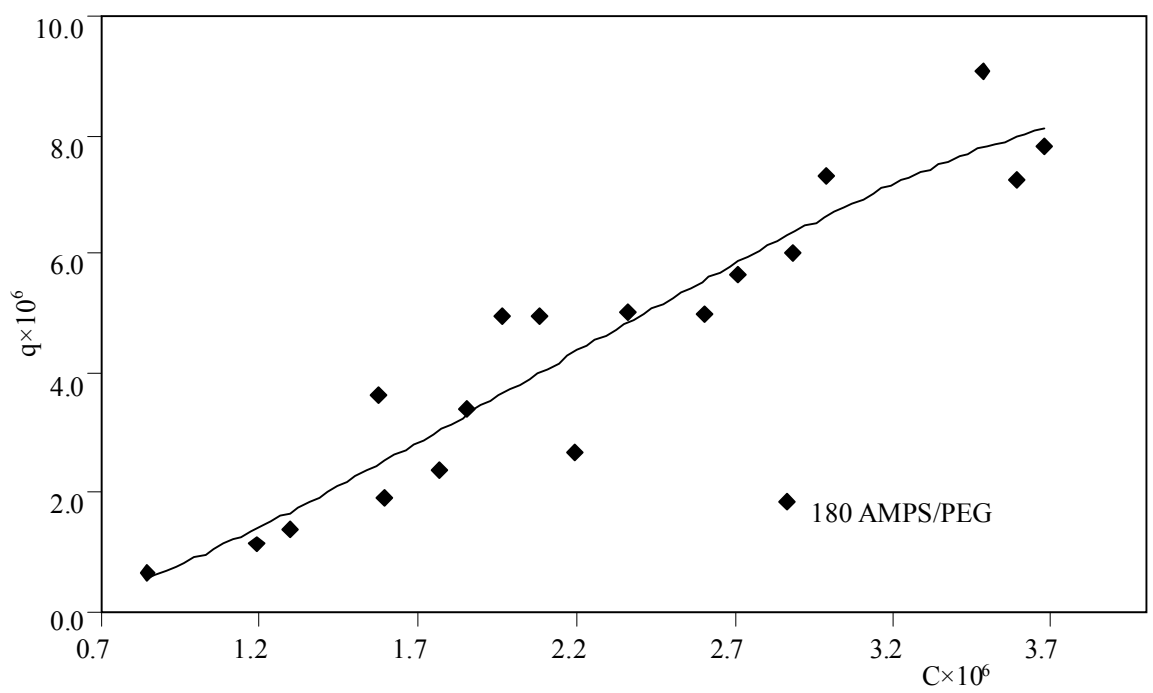

Figure 11. Equilibrium sorption isotherms of AAm/AMPS/PEG hydrogels in aqueous LV solutions.

studies. Both of them, it can be seen that swelling or sorption capability of AAm/AMPS hydrogels and Aam /AMPS/PEG semi IPNs are increased with increasing AMPS content in copolymeric structure. The most important effect is hydrophilicity of copolymeric gels. Hydrophilicity of AAm/AMPS and AAm/AMPSA/PEG copolymers becomes greater than that of AAm, when addition of AMPS to the copolymeric structure.

There can be many reasons for non-covalent interactions in the binding of LV by AAm/AMPS hydrogels and AAm/AMPS/PEG semi IPNs. The main interactions between the hydrogel and the monovalent cationic dye may be hydrophobic and hydrogen bonding. Specially, hydrogen bonding will be expected to occur between amine groups and nitrogen atoms on the dye molecules and the amine and carbonyl groups on the monomer unit of crosslinked polymer. Hydrophobic effects are especially aqueous solutions interactions which in the present case will involve those aromatic rings on the dye molecules and the methine and methyl groups on the gel. There can be some other interactions such as dipole-dipole and dipole-induced dipole interactions between the dye molecules and the hydrogel chains.

\subsection{PEG Effect on the Sorption of LV}

For investigation of the effect of mass/content of PEG on the sorption properties of AAm/AMPS/PEG semi IPNs, some sorption parameters such as dye removal capacity, adsorption percentage and partition coefficient of AAm/AMPS/PEG semi IPN systems containing 180 mg AMPS are tabulated in Table 6.

It was shown that a increasing of adsorption percentage $(90.30 \%-94.70 \%)$ and partition coefficient (9.31 17.87) of AAm/AMPS/PEG semi IPN systems when PEG has been added to the hydrogel systems. Incorpo- 
ration of PEG into the copolymer network leads to higher values of adsorption percentage and partition coefficient of AAm/AMPS/PEG semi IPN systems. Here, $\mathrm{K}_{\mathrm{d}}$ values of AAm/AMPS/PEG semi IPNs are higher than 1.0. So, it can be said that synthesized crosslinked AAm/AMPS /PEG semi IPNs could be used as potential water adsorbent $[38,39]$. On the other hand, it can be said that there is no important chancing of the dye removal capacity of AAm/AMPS/PEG semi IPN systems when PEG has been added to the hydrogel systems $\left(0.94 \times 10^{-5}-1.08 \times\right.$ $\left.10^{-5} \mathrm{~mol} \mathrm{~g}^{-1}\right)$. Here, it was said that PEG chains was placed in the crosslinked polymeric systems, in stead of crosslinked AAm and AMPS molecules, it was seen that increasing of the adsorption percentage, because of increasing of hydrophilic character at crosslinked polymeric systems.

\section{Conclusion}

Incorporation of hydrophilic group containing chemicals such as AMPS and a polymer such as PEG in AAm hydrogels can be obtained successively by free radical solution polymerization method. Multifunctional crosslinker such as TMPTA used at the polymerization process. AAm/AMPS hydrogels and AAm/AMPS/ PEG semi IPNs showed high water absorbency. The equilibrium percentage swelling ranges are 900\% - 13450\% for AAm/AMPS hydrogels and 500\% - 4400\% for AAm /AMPS/PEG semi IPNs. It was seen that swelling of AAm/AMPS hydrogels and AAm/AMPS/ PEG semi IPNs increased with the increasing of content of AMPS. But, it was seen that a decreasing of values of Seq\% from $3150 \%$ to $1500 \%$ when the adding of PEG for containing of $180 \mathrm{mg}$ of AMPS. It was seen that swelling values of AAm/AMPS hydrogels and AAm/AMPS/PEG semi IPNs with increasing in aqueous $0.01 \mathrm{M} / 0.03 \mathrm{M}$ urea solutions. Also, it was seen that a increasing of values of $\mathrm{S}_{\mathrm{eq}} \%$ from $3150 \%$ (for water) to $4350 \%-4440 \%$ when the adding of PEG for containing of $180 \mathrm{mg}$ of AMPS. Here, again, the main effect is the hydrophilic character of AMPS and urea molecules.

The present work has given the quantitative information on the sorption characteristic of LV with AAm /AMPS hydrogels and AAm/AMPS/PEG semi IPNs. In this study, it has shown that AAm/AMPS hydrogels and AAm/AMPS/PEG semi IPNs have sorbed the monovalent cationic dyes such as LV, while AAm do not. The amount of LV sorbed onto unit dry mass of the gel is increased with the content of AMPS. The values of adsorption percentage $(\mathrm{Ads} \%)$ for LV of AAm/AMPS hydrogels and AAm/AMPS/PEG semi IPNs are changed among $71.75 \%$ - 96.31\%. Here, all Kd value of AAm /AMPS hydrogels and AAm/AMPS/PEG semi IPNs is higher than 1.0. So, it can be said that synthesized crosslinked AAm/AMPS hydrogels and AAm/AMPS /PEG semi IPNs could be used as potential highly water adsorbent. Consequently, AAm/AMPS hydrogels and AAm/AMPS/PEG semi IPNs developed in this study may serve as a potential device for water and dye sorbent. The utilization of these types of materials, in pharmaceuticals, agriculture, biotechnology, environment, separation, purification and immobilization makes hydrogels more popular.

\section{References}

[1] S. Li, "Removal of Crystal Violet from Aqueous Solution by Sorption into Semi-Interpenetrated Networks Hydrogels Constituted of Poly (Acrylic Acid-AcrylamideMethacrylate) and Amylose," Bioresource Technology, Vol. 101, 2010, pp. 2197-2202. doi:10.1016/j.biortech.2009.11.044

[2] G. Güçlü, E. Al, S. Emik, T. B. İyim, S. Özgümüş and M. Özyürek, "Removal of $\mathrm{Cu}^{2+}$ and $\mathrm{Pb}^{2+}$ Ions from Aqueous Solutions by Starch-Graft-Acrylic Acid/ Montmorillonite Superabsorbent Nanocomposite Hydrogels," Polymer Bulletin, Vol. 65, 2010, pp. 333-346. doi:10.1007/s00289-009-0217-x

[3] A. L. Buyanov, I. V. Gofman, L. G. Revel'skaya, A. K. Khripunov and A. A. Tkachenko, "Anisotropic Swelling and Mechanical Behavior of Composite Bacterial Cellulose-Poly (aAcrylamide or Acrylamide-Sodium Acrylate) Hydrogels," Journal of the Mechanical Behavior of Biomedical Materials, Vol. 3, 2010, pp. 102-111. doi:10.1016/j.jmbbm.2009.06.001

[4] E. Karadağ, T. Kırıştı, S. Kundakcı and Ö. B. Üzüm, "Investigation of Sorption/Swelling Characteristics of Chemically Crosslinked AAm/SMA Hydrogels as Biopotential Sorbent," Journal of Applied Polymer Science, Vol. 117, No. 3, 2010, pp. 1787-1797.

[5] E. Uğuzdoğan, E. B. Denkbaş and O. S. Kabasakal, "The Use of Polyethyleneglycolmethacrylate-Covinylimi-dazole (PEGMA-co-VI) Microspheres for the Removal of Nickel(II) and Chromium(VI) Ions," Journal of Hazardous Materials, Vol. 177, 2010, pp. 119125.

doi:10.1016/j.jhazmat.2009.12.004

[6] E. Karadağ, S. Kundakcı and Ö. B. Üzüm, "Water Sorption and Dye Uptake Studies of Highly Swollen AAm/AMPS Hydrogels and Semi-IPN's with PEG," Polymer-Plastics Technology and Engineering, Vol. 48, No. 12, 2009, pp. 1217-1229.

[7] N. Şahiner, "Colloidal Nanocomposite Hydrogel Particles," Colloid and Polymer Science, Vol. 285, 2007, pp. 413-421. doi:10.1007/s00396-006-1583-7

[8] B. Chu and B. S. Hsiao, "The Role of Polymers in Breakthrough Technologies for Water Purification," Journal of Polymer Science Part B: Polymer Physics, Vol. 47, 2009, pp. 2431-2435. doi:10.1002/polb.21854 
[9] M. Ç. Akkaya, S. Emik, G. Güçlü, T. B. İyim and S. Özgümüş, "Removal of Basic Dyes from Aqueous Solutions by Crosslinked-Acrylic Acid/Acrylamidopropane Sulfonic Acid Hydrogels," Journal of Applied Polymer Science, Vol. 114, 2009, pp. 1150-1159. doi:10.1002/app.30704

[10] Ö. Özay, S. Ekici, Y. Baran, N. Aktaş and N. Şahiner, "Removal of Toxic Metal Ions with Magnetic Hydrogels," Water Research, Vol. 43, 2009, pp. 4403-4411. doi:10.1016/j.watres.2009.06.058

[11] V. Bekiari, M. Sotiropoulou, G. Bokias and P. Lianos, "Use of Poly (N,N-Dimethylacrylamide-Co-Sodium Acrylate) Hydrogel to Extract Cationic Dyes and Metals from Water," Colloids and Surfaces A: Physicochemical and Engineering Aspects, Vol. 312, 2008, pp. 214-218. doi:10.1016/j.colsurfa.2007.06.053

[12] E. Karadağ, Ö. B. Üzüm, S. Kundakcı and D. Saraydın, "Polyelectrolyte CASA Hydrogels for Uptake of Uuranyl Ions from Aqueous Solutions," Journal of Applied Polymer Science, Vol. 104, 2007, pp. 200-204. doi:10.1002/app.25397

[13] E. K. Yetimoğlu, M. V. Kahraman, Ö. Ercan, Z. S. Akdemir and N. Kayaman Apohan, "N. N-vinylpyyrolidone /Acrylic Acid/2-Aacrylamido-2-Methylpropane Sulponic Acid Based Hydrogels: Synthesis, Characterization and Their Application in the Removal of Heavy Metals," Reactive Functional Polymers, Vol. 67, 2007, pp. 451-460. doi:10.1016/j.reactfunctpolym.2007.02.007

[14] N. Şahiner, W. T. Godbey, G. L. McPherson and V. T. John, "Microgel, Nanogel and Hydrogel-Hydrogel SemiIPN Composites for Biomedical Applications: Synthesis and Characterization," Colloid and Polymer Science, Vol. 284, 2006, pp. 1121-1129. doi:10.1007/s00396-006-1489-4

[15] G. Crini, "Recent dDevelopments in PolysaccharideBased Materials Used as Adsorbents in Wastewater Treatment," Progress Polymer Science, Vol. 30, 2005, pp. 38-70. doi:10.1016/j.progpolymsci.2004.11.002

[16] G. Crini, "Non-Conventional Low-Cost Adsorbents for the Removal: A Review," Bioresource Technology, Vol. 97, 2006, pp. 1061-1085. doi:10.1016/j.biortech.2005.05.001

[17] E. Karadağ, Ö. B. Üzüm and D. Saraydın, "Swelling Equilibria and Dye Adsorption Studies of Chemically Crosslinked Superabsorbent Acrylamide/Maleic Acid Hydrogels," European Polymer Journal, Vol. 38, 2002, pp. 2133-2141. doi:10.1016/S0014-3057(02)00117-9

[18] O. Güven, M. Şen, E. Karadağ and D. Saraydın, “A Review on the Radiation Synthesis of Copolymeric Hydrogels for Adsorption and Seperation Purposes," Radiation Physics and Chemistry, Vol. 56, 1999, pp. 381-386. doi:10.1016/S0969-806X(99)00326-6

[19] L. Serra, J. Domenech and N. A. Peppas, "Design of Poly (Ethylene Glycol)-Tethered Copolymers as Novel Mucoadhesive Drug Delivery System," European Journal of Pharmaceutics and Biopharmaceutics, Vol. 63, 2006, pp. 11-18. doi:10.1016/j.ejpb.2005.10.011
[20] P. Krsko and M. Libera, "Biointeractive Hydrogels," Materials Today, Vol. 8, 2005, pp. 36-44. doi:10.1016/S1369-7021(05)71223-2

[21] J. D. Kosmala, D. B. Henthorn and L. Brannon-Peppas, "Preparation of Interpenetrating Network of Gelatin and Dextran as Degradable Biomaterials," Biomaterials, Vol. 21, 2002, pp. 2019-2023. doi:10.1016/S0142-9612(00)00057-0

[22] D. Chakrabarty, "Interpenetrating Polymer Networks: Engineering Properties and Morphology," Polymer Gels and Networks, Vol. 6, 1998, pp. 191-204. doi:10.1016/S0966-7822(98)00011-2

[23] J. P. Chen and S. H. Chiu, "A Poly ( $N$-Isopropylacrylamide - $\mathrm{CO}-\mathrm{N}$-Acryloxysuccinimide-Co-2-Hydroxyethyl Methacrylate) Composite Hydrogel Membrane for Urease Immobilization to Enhance Urea Hydrolysis Rate by Temperature Swing," Enzyme and Microbial Technology, Vol. 26, 2000, pp. 359-367. doi:10.1016/S0141-0229(99)00181-7

[24] Z.Tong, L. Yuhai, Y. Shihuo and H. Zhongyi, "Superabsorbent Hydrogels as Carriers for the Controlled-Release of Urea: Experiments and a Mathematical Model Describing the Release Rate," Biosystems Engineering, Vol. 102, 2009, pp. 44-50. doi:10.1016/j.biosystemseng.2008.09.027

[25] E. Karadağ, Ö. B. Üzüm, D. Saraydın and O. Güven, "Dynamic Swelling Behavior of $\gamma$-Radiation Induced Polyelectrolyte Poly(AAm-co-CA) Hydrogels in Urea Solutions," Interanational Journal of Pharmaceutics, Vol. 301, 2005, pp. 102-111. doi:10.1016/j.ijpharm.2005.05.026

[26] E. Karadağ, Ö. B. Üzüm, D. Saraydın and O. Güven, "Swelling Characterization of $\gamma$-Radiation Induced CAMA Hydrogels in Urea Solutions," Materials Design, Vol. 27, No. 7, 2006, pp. 576-584. doi:10.1016/j.matdes.2004.11.019

[27] A. Martinez-Ruvalcaba, J. C. Sanchez-Diaz, F. Becerra and L. E. Cruz-Barba, "Swelling Characterization and Drug Delivery Kinetics of Polyacrylamide-Co-Itaconic Acid/Chitosan Hydrogels," Express Polym Letter, Vol. 3, 2009, pp. 25-32. doi:10.3144/expresspolymlett.2009.5

[28] Y. M. Mohan, P. S. K. Murthy and K. M. Raju, "Preparation and Swelling Behavior of Macroporous Poly (Acrylamide-Co-Sodium Methacrylate) Superabsorbent Hydrogels," Journal of Applied Polymer Science, Vol. 101, 2006, pp. 3202-3214. doi:10.1002/app.23277

[29] E. Karadağ and Ö. B. Üzüm, "Sorption for Removing Lauths Violets in Aqueous Solutions by Chemically Crosslinked Poly (AAm-co-SA) Hydrogels," Polymer Bulletin, Vol. 53, 2005, pp. 387-392. doi:10.1007/s00289-005-0351-z

[30] S. Durmaz and O. Okay, "Acrylamide/2-Acrylamido-2Methylpropanesulfonic Acid Sodium Salt-Based Hydrogels: Synthesis and Characterization," Polymer, Vol. 41, 2000, pp. 3693-3704. doi:10.1016/S0032-3861(99)00558-3 
[31] S. Çavuş and G. Gürdağ, "Competitive Heavy Metal Removal by Poly/2-Acrylamido-2-Methyl-1-Propane Sulfonic Acid-Co-Itaconic Acid)," Polymers for Advanced Technologies, Vol. 19, 2008, pp. 1209-1217. doi:10.1002/pat.1113

[32] S. Kundakc1, Ö. B. Üzüm and E. Karadağ, "A New Composite Sorbent for Water and Dye Uptake: Highly Swollen Acrylamide/2-Acrylamido-2-Methyl-1-Propan-esulfonic Acid/Clay Hydrogels Crosslinked by 1, 4-Butane-diol Dimeth-Acrylate," Polymer Composites, Vol. 30, 2009, pp. 29-37. doi:10.1002/pc.20524

[33] D. Saraydın, E. Karadağ, Y. Işıkver, N. Şahiner and O. Güven, "The Influence of Preparation Methods on the Swelling and Network Properties of Acrylamide Hydrogels with Crosslinkers," Journal of Macromoleculer Science, Part A - Pure and Applied Chemistry,Vol. A41, No. 4, 2004, pp. 419-431.

[34] S. J. Kim, S. J. Park and S. I. Kim, "Synthesis and Characterization of Interpenetrating Polymer Network Hydrogels Composed of Poly(Vinyl Alcohol) and Poly ( $\mathrm{N}$ Isopropylacrylamide)," Reactive Functional Polymers, Vol. 55, 2003, pp. 61-67. doi:10.1016/S1381-5148(02)00215-8

[35] N. A. Peppas and N. M. Franson, "The Swelling Interface Number as a Criterion for Prediction of Diffusional Sol- ute Release Mechanisms in Swellable Polymers," Journal of Polymer Science Polymer Physics Edition, Vol. 21, 1983, pp. 983-997. doi:10.1002/pol.1983.180210614

[36] T. Çaykara, S. Kiper and G. Demirel, "Thermosensitive Poly (N-Isopropilacrylamide-Co-Acrylamide) Hydrogels: Synthesis, Swelling and Interaction with Ionic Surfactants," European Polymer Journal, Vol. 42, 2006, pp. 348-355. doi:10.1016/j.eurpolymj.2005.07.006

[37] R. Dengre, M. Bajpai and S. K. Bajpai, "Release of Vitamin B12 from Poly (N-Vinyl-2-Pyrrolidione)-Crosslinked Polyacrylamide Hydrogels," Journal of Applied Polymer Science, Vol. 76, 2000, pp. 1706-1714. doi:10.1002/(SICI)1097-4628(20000613)76:11<1706:: AID-APP12>3.0.CO;2-W

[38] L. M. Schwarte and N. A. Peppas, "Novel Poly (Ethylene Glycol)-Grafted Cationic Hydrogels: Preparation, Characterization and Diffusive Properties," Polymer, Vol. 39, 1998, pp. 6057-6066. doi:10.1016/S0032-3861(98)00087-1

[39] N. Şahiner, D. Saraydın, E. Karadağ and O. Güven, "Swelling and Dye Adsorption Properties of Radiation Induced N-Vinyl-2-Pyrrolidone/Acrylonitrile Hydroge1s," Polymer Bulletin, Vol. 41, No. 3, 1998, pp. 371-378. doi:10.1007/s002890050376 